Research Paper

\title{
Establishing a three-miRNA signature as a prognostic model for colorectal cancer through bioinformatics analysis
}

\author{
Yiming Wang ${ }^{1,}{ }^{*}$, Lumi Huang ${ }^{1,},{ }^{*}$ Nan Shan ${ }^{2}$, Huiwen Ma ${ }^{1}$, Songmei Lu ${ }^{1}$, Xingyue Chen ${ }^{1}$, Hao Long ${ }^{3}$ \\ ${ }^{1}$ Department of Medical Oncology, Chongqing University Cancer Hospital, Chongqing, China \\ ${ }^{2}$ Department of Gynaecology and Obstetrics, The First Affiliated Hospital of Chongqing Medical University, \\ Chongqing, China \\ ${ }^{3}$ Department of Palliative Care, Chongqing University Cancer Hospital, Chongqing, China \\ *Equal contribution
}

Correspondence to: Hao Long; email: lyq9167@163.com, https://orcid.org/0000-0002-3008-655X

Keywords: CCa, miRNA, risk score, prognostic model

Received: March 3, $2021 \quad$ Accepted: July 30, 2021

Published: August 13, 2021

Copyright: $(2021$ Wang et al. This is an open access article distributed under the terms of the Creative Commons Attribution License (CC BY 3.0), which permits unrestricted use, distribution, and reproduction in any medium, provided the original author and source are credited.

\section{ABSTRACT}

Background: Identification of more promising microRNAs (miRNAs) are being extensively studied with respect to colorectal cancer (CRC), since CRC is the leading cause of cancer deaths and most common malignant tumors worldwide. A series of colon cancer (CCa) samples from The Cancer Genome Atlas (TCGA) were analyzed to provide a new perspective into this field.

Methods: The expression of miRNAs, mRNAs and the clinical data of 437 CRC patients were downloaded from the TCGA database. The survival-related differentially expressed miRNAs (sDMIRs) and mRNAs were detected by COX regression analysis. The high-risk group and low-risk group were separated by the median risk score of the risk score model. The potential clinical characteristics of these sDMIRs were analyzed by $R$ software. The potential molecular mechanisms of these SDMIRs were explored by computational biology. The expression levels of three sDMIRs were explored by qPCR in CRC samples.

Results: Three DMIRs (hsa-miR-21-3p, hsa-miR-194-3p and hsa-miR-891a-5p) correlated with the most remarkable prognostic values of CRC patients were selected to establish the risk score model (RSM) by univariate and multivariate COX regression analysis and the survival probability of the low-risk group was longer than that in the high-risk group. We detected the target genes of three sDMIRs and the potential molecular mechanisms of these sDMIRs. We further verified the high expression levels of hsa-miR-21-3p and hsa-miR-194-3p were associated with the early T-stages, while hsa-miR-891a-5p illustrated the reversed result. Conclusion: Our study demonstrated three SDMIRs with significantly clinical values illustrated the potential predicting values in the prognosis of CRC patients. Our results may provide a new perspective for the diagnostic methods and treatment strategies in CRC patients.

\section{INTRODUCTION}

Colorectal cancer (CRC) is one of the most common malignant tumors and causes the second most cancer deaths worldwide [1,2]. At present, surgery combined with adjuvant therapies such as chemotherapy and radiotherapy is the primary strategy. However, the 5year postoperative survival rate of CRC patients is less than $30 \%$ [3, 4]. Therefore, increasing numbers of studies try to explore promising predicting models which can help to identify CRC patients with considerable sensitivity earlier.

With the more insightful recognition of the importance of microRNAs (miRNAs), a class of approximate 20 nucleotide-long, double-stranded, 
non-coding RNAs (ncRNA), increasing numbers of studies have demonstrated miRNAs not only take part in post-transcriptional modifications, but also play critical regulating roles on anticancer immune responses and predicting prognosis [5, 6]. MiRNAs are potential for amending tumor cells behaviors due to abnormal gene profiles for mature and precursor sequences compared to normal tissues [7, 8]. Consisting of different tumor stromal cells, abundant ncRNA and other cytokines, tumor microenvironment (TME) is the pivotal concern of identifying prognosisrelated markers $[9,10]$. In the past dozen years, miRNAs have been demonstrated to be closely associated with the occurrence, development and prognosis of different tumors $[11,12]$. For example, miR-21-3p has been demonstrated to be high expressed in gastric cancer, pheochromocytoma, esophageal cancer and other malignant tumors [1315]. The attenuation of miR-17 also regulates cell death and mediates expression of SRC-3 and CLU in CRC cells [16]. MiR-194-3p highly expressed in various malignant tumors such as nasopharyngeal carcinoma, endometrial cancer and pancreatic cancer [17-19]. Besides, miR-891a-5p was found low expressed in breast cancer [20]. Therefore, we found miRNAs as a type of biomarkers in the TME, exhibit potential for predicting therapeutic sensitivity and prognosis. However, the roles of miR-21-3p, miR194-3p and miR-891a-5p in CRC remain unclear, and few reports illuminated the clinical significance of miR-21-3p, miR-194-3p and miR-891a-5p.

The present study attempts to the clinical significance of miRNAs, especially in assessing the prognoses of CRC patients. The findings will help to open up new avenues for discovering the underlying mechanisms of miRNAs in CRC, and the RSM will also provide a novel perspective for clinical strategy making.

\section{MATERIALS AND METHODS}

\section{Clinical colon samples}

168 colon tumor tissues and adjacent normal tissues were obtained from the patients who performed operation in Chongqing University Cancer Hospital from September 2018 to March 2020. The obtained samples were frozen in liquid nitrogen immediately and then stored at $-80^{\circ} \mathrm{C}$ until miRNAs extraction.

\section{Ethics approval and consent to participate}

All patients signed the informed consent forms. The research protocol abides by the ethical principles of medical research and is approved by the Ethics Committee of Chongqing University Cancer Hospital.

\section{Data acquisition and differentially expressed analysis}

We downed the transcriptome mRNAs and miRNAs data of CRC from The Cancer Genome Atlas (TCGA) data portal (https://portal.gdc.cancer.gov/). The data in the present study were currently updated on May 7, 2020. We excluded the data of patients with the overall survival (OS) of CRC patients $\leq 30$ days, because these patients probably died from other causes). We then acquired the genes with the differential expression in tumor tissues and adjacent normal tissues by the limma package of $\mathrm{R}$ software (https://bioconductor.org/ packages/release/bioc/html/limma.html). The screening value of all differentially expressed mRNAs and miRNAs is "FDR $<0.05, \log _{2}|\mathrm{FC}|>1$ and $\mathrm{P}<0.05$ ".

\section{Survival-related differentially expressed miRNAs (sDMIRs) and the risk score model (RSM)}

Differentially expressed miRNAs (DMIRs) with significant clinical outcomes were served as survivalrelated DMIRs (sDMIRs). We utilized univariate COX regression analysis to screen sDMIRs $(P<0.001)$ and the value of hazard ratio confirmed sDMIRs into protective and deleterious parts. The sDMIRs were further analyzed by the multivariate $\mathrm{COX}$ regression analysis, and were employed to establish the RSM. RSM was built by the expression levels multiplied by Cox regression coefficients. Based on the median risk score of RSM, CRC patients were separated into the high-risk group and the low-risk group. We further analyzed the relevance of the RSM and clinical features by T-test to detect the clinical application value of the sDMIRs.

\section{Real-time quantitative PCR}

The PrimeScript RT reagent kit (TaKaRa, Osaka, Japan) was used to reverse-transcribe RNAs to cDNAs. The reaction steps [21] were shown as follows: $37^{\circ} \mathrm{C}$ for $15 \mathrm{~min}$ and $85^{\circ} \mathrm{C}$ for $5 \mathrm{~s}$. The quantitative polymerase chain reaction (qPCR) was run on an $\mathrm{ABI}$ 7500 Real-Time PCR System (Applied Biosystems) using a SYBR Green assay (TaKaRa). The reaction cycling conditions were $95^{\circ} \mathrm{C}$ for $30 \mathrm{~s}, 40$ cycles of $195^{\circ} \mathrm{C}$ for $5 \mathrm{~s}$, and $60^{\circ} \mathrm{C}$ for $34 \mathrm{~s}$; primer sequences are shown in Table 1. The relative quantification levels of miRNAs were standardized to U6 by the $2^{-\Delta \mathrm{Ct}}$ method. Each cDNA sample was replicated three assays.

\section{Bioinformatics analysis}

We employed the miRTarBase (https://mirtarbase. cuhk.edu.cn/ miRTarBase/miRTarBase_2019/php/inde x.php), TargetScan (http://www.targetscan.org/vert 72/) and miRDB (http://mirdb.org/) databases to screen the target genes (TGs). A target gene needs to meet a 
Table 1. The primer sequences of hsa-miR-21-3p, hsa-miR-194-3p, and hsa-miR-891a-5p.

\begin{tabular}{|c|c|c|}
\hline \multirow{4}{*}{ hsa-miR-21-3p } & F primer( $\left.5^{\prime}-3^{\prime}\right)$ & GACCCAACACCAGTCGATG \\
\hline & $\mathrm{R}$ primer( $\left.5^{\prime}-3^{\prime}\right)$ & тССТССТСТССТТССТТСТС \\
\hline & $\mathrm{RT}\left(5^{\prime}-3^{\prime}\right)$ & $\begin{array}{l}\text { GTCCTCСТCTCCTTCCTTCTCATGAGGAGGACACA } \\
\text { GCC }\end{array}$ \\
\hline & F primer( $\left.5^{\prime}-3^{\prime}\right)$ & CCAGTGGGGCTGCTGT \\
\hline \multirow{2}{*}{$\begin{array}{l}\text { hsa-miR-194- } \\
\text { 3p }\end{array}$} & $\mathrm{R}$ primer( $\left.5^{\prime}-3^{\prime}\right)$ & GAGAGGAGAGGAAGAGGGAA \\
\hline & $\mathrm{RT}\left(5^{\prime}-3^{\prime}\right)$ & $\begin{array}{l}\text { GGAGAGGAGAGGAAGAGGGAAATCTCCTCTCCC } \\
\text { AGATA }\end{array}$ \\
\hline \multirow{4}{*}{$\begin{array}{l}\text { hsa-miR-891a- } \\
5 p\end{array}$} & F primer( $\left.5^{\prime}-3^{\prime}\right)$ & GTGCAACGAACCTGAGC \\
\hline & $\mathrm{R}$ primer( $\left.5^{\prime}-3^{\prime}\right)$ & GACCTGAACCTGAACCTGAA \\
\hline & $\mathrm{RT}\left(5^{\prime}-3^{\prime}\right)$ & $\begin{array}{l}\text { GGACCTGAACCTGAACCTGAAATTTCAGGTCCTC } \\
\text { AGTG }\end{array}$ \\
\hline & F primer( $\left.5^{\prime}-3^{\prime}\right)$ & CTCGCTTCGGCAGCACA \\
\hline \multirow{2}{*}{ U6 } & $\mathrm{R}$ primer( $\left.5^{\prime}-3^{\prime}\right)$ & AACGCTTCACGAATTTGCGT \\
\hline & RT(5'-3') & AAAATATGGAACGCTTCACGAATTTG \\
\hline
\end{tabular}

Note: F primer, forward primer; R primer, reverse primer; RT, reverse transcription.

standard of two databases at least. In order to further investigate these TGs, the GO and KEGG pathways were employed to discover the underlying mechanisms. The receiver operating characteristic (ROC) curve was generated by the $\mathrm{R}$ software. Univariate and multivariate Cox regression analysis were employed to confirm the sDMIRs. The survival probability of CRC patients was assessed by KaplanMeier survival curve.

\section{Statistical analysis}

GraphPad Prism5 (GraphPad Software Inc, La Jolla, CA, USA) and SPSS19.0 software (SPSS Inc, Chicago, IL, USA) were utilized for statistical analyses. The clinical correlations were determined by ANOVA, posthoc test (Boferroni method) and independent T-test. $P<$ 0.05 was considered statistically significant.

\section{Availability of data and materials}

Authors can provide all of datasets analyzed during the study on reasonable request.

\section{RESULTS}

\section{MiRNAs and mRNAs with differential expression}

Through limma algorithm, we identified 496 differentially expressed miRNAs (DMIRs), of which 212 were downward and 285 were raised (Figure 1A and Supplementary Table 1). And the 10 most downward and raised miRNAs were illustrated in the heatmap by the value of $\log _{2}|\mathrm{FC}|$ (Figure 1B). Next, 1533 CRC genes with differential expression (DMRs) were also identified, including 651 downward and 882 raised mRNAs (Figure 1C). The 10 most downward and raised mRNAs were shown in Figure 1D.

\section{The relevancies between DMIRs and clinical prognosis}

We selected 3 DMIRs which were associated with the clinical prognoses of CRC patients (sDMIRs), including hsa-miR-194-3p, hsa-miR-21-3p and hsa-miR-891a-5p. The relationships between these sDMIRs and clinical prognosis were shown in Figure 2, of which has-miR891a-5p showed a positive relation with the poor clinical prognosis, but hsa-miR-194-3p and hsa-miR-21-3p were illustrated negative correlations with the poor clinical prognosis. Three sDMIRs (hsa-miR-891a-5p, hsa-miR21-3p and hsa-miR-194-3p) were identified by the multivariate $\mathrm{COX}$ regression analysis to establish the risk score model (RSM). The formula was as followed, [Expression levels of hsa-miR-891a-5p* (0.190971)] + [Expression of hsa-miR-194-3p * $(-0.220654)]+$ [Expression of hsa-miR-21-3p * (-0.453552)]. As shown in the survival curve, the high level of hsa-miR-891a-5p indicated the poor prognosis, while the hsa-miR-21-3p and hsa-miR-194-3p showed positive relations with OS (Supplementary Figure 1).

\section{Clinical features and application of RSM}

The sDMIRs were used to establish RSM, and the CRC patients were divided into the low-risk group and the high-risk group (Figure 3A). As illuminated in the Figure $3 \mathrm{~B}$, the mortality decreased with the lower risk score. Besides, with the increased risk score, the expression of hsa-miR-891a-5p rather than hsa-miR-21$3 p$ and hsa-miR-194-3p was prominently enhanced 
A

Volcano

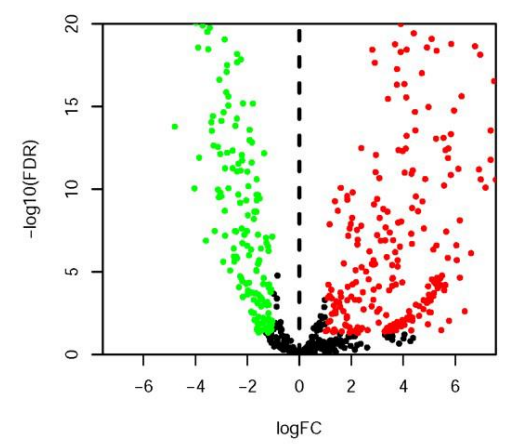

C

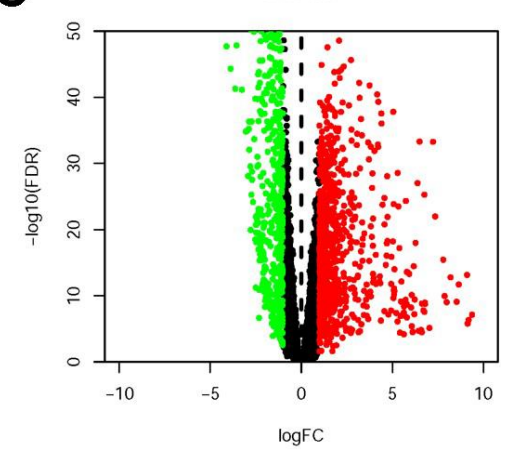

B

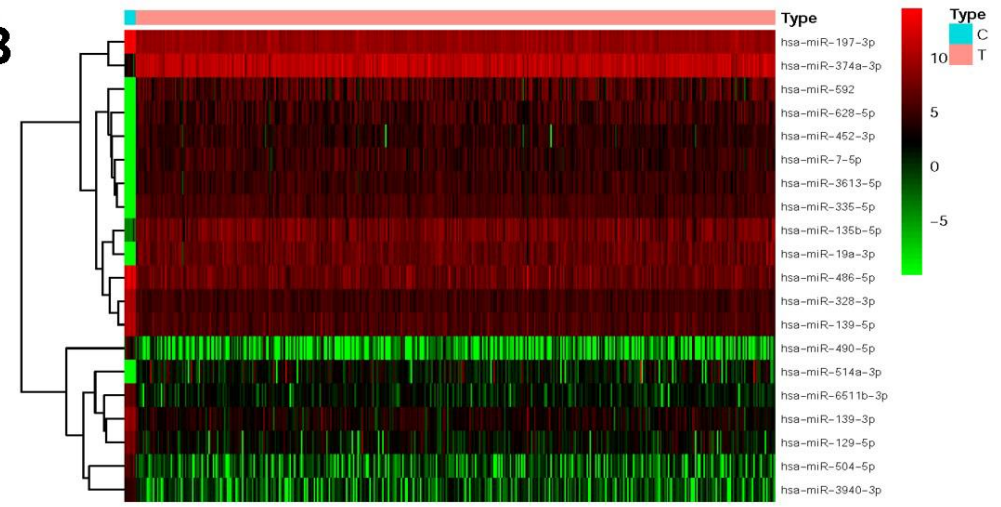

D

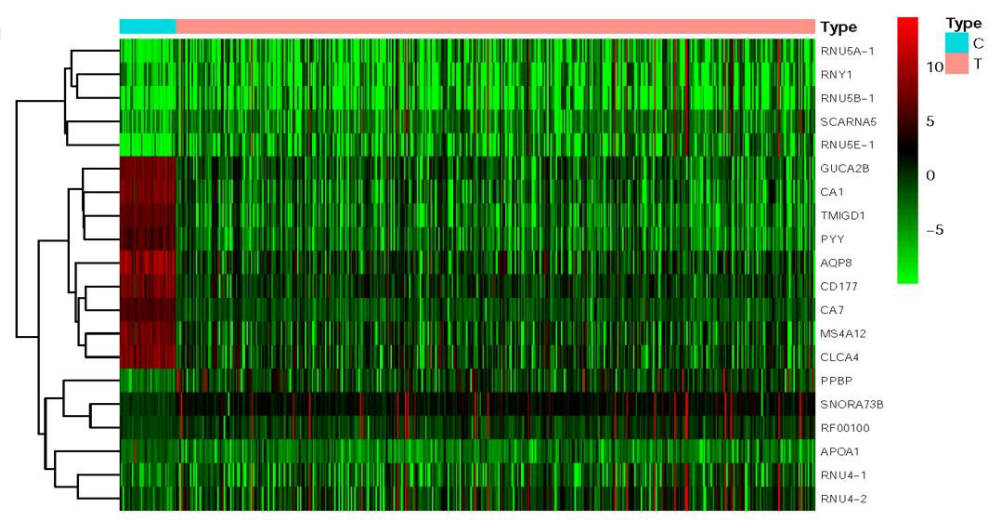

Figure 1. Differentially expressed miRNAs and mRNAs in CRC. The differentially expressed miRNAs (DMIRs) in CRC and adjacent nontumor tissues were showed in the volcano plot (A) and heatmap (B) based on TCGA database. Volcano plot (C) and heatmap (D) demonstrated the differentially expressed mRNAs between CRC tissues and adjacent normal tissues based on TCGA database. The red parts represented the upregulated genes; the green parts represented the downregulated genes, and the black parts represented the genes without significant difference. $\mathrm{FDR}<0.05, \log _{2}|\mathrm{FC}|>1$ and $P<0.05$.

pvalue

hsa-miR-194-3p $\quad<0.001$

$0.717(0.590-0.870)$

hsa-miR-21-3p $\quad<0.001$

hsa-miR-891a-5p $\quad<0.001$

Hazard ratio

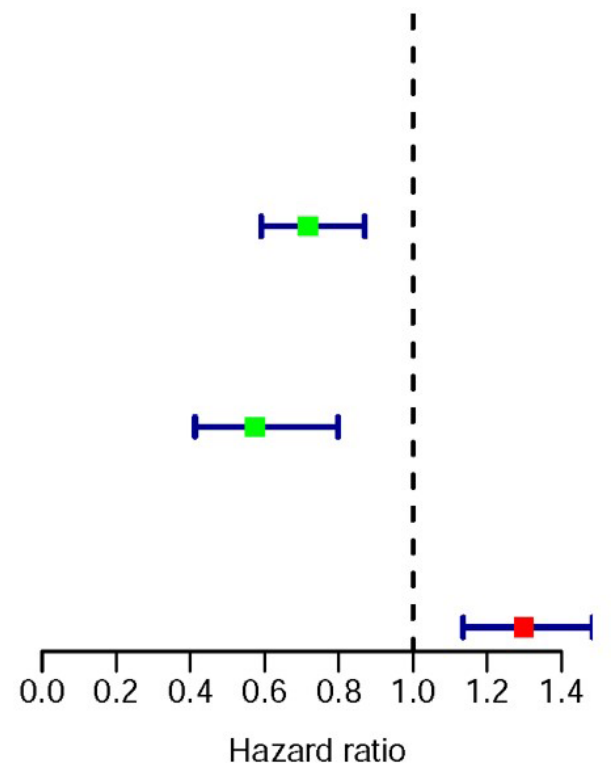

Figure 2. DMIRs with significant clinical outcomes. The forest plot showed the survival-related hazard ratio of sDMIRs (hsa-miR-194-3p, hsa-miR-21-3p and hsa-miR-891a-5p) based on TCGA database. Red parts represented the up-regulation, and green parts represented the down-regulation. 
(Figure 3C). The high-risk group showed the poorer OS than the low-risk group (Figure 4A). The ROC curve was employed to investigate the accuracy of RSM, and the area under curve of the ROC curve was 0.713 , meaning the RSM in survival prediction was satisfied (Figure 4B). To further investigate the clinical application of RSM and the sDMIRs, we detected the relevance between the RSM and the clinical features, such as age, gender, and TNMstage. We found that female (Figure 5A), advanced stage (Figure 5B) and advanced TNM-stage (Figure 5C-5E) were the remarkable risk factors with the higher scores. Next, we further analyzed the relationships between sDMIRs and the clinical features. We found that the early young (Figure 6A), advanced stage (Figure 6B), and advanced TNM-stage (Figure 6C-6E) were correlated with the lower expression level of hsa-miR-21-3p. Besides, hsa-miR-194-3p expressed decreasingly with the advanced stage (Figure 6F) and advanced NM-stage (Figure 6G, 6H). The male patients (Figure 6I) and patients with early T-stage (Figure 6J) showed the negative correlations with the increased expression level of hsa-miR-891a-5p.

\section{The TGs of the sDMIRs and functional enrichment analysis}

In order to detect the TGs of sDMIRs, we predicted and screened the TGs by the databases of TargetScan,

A
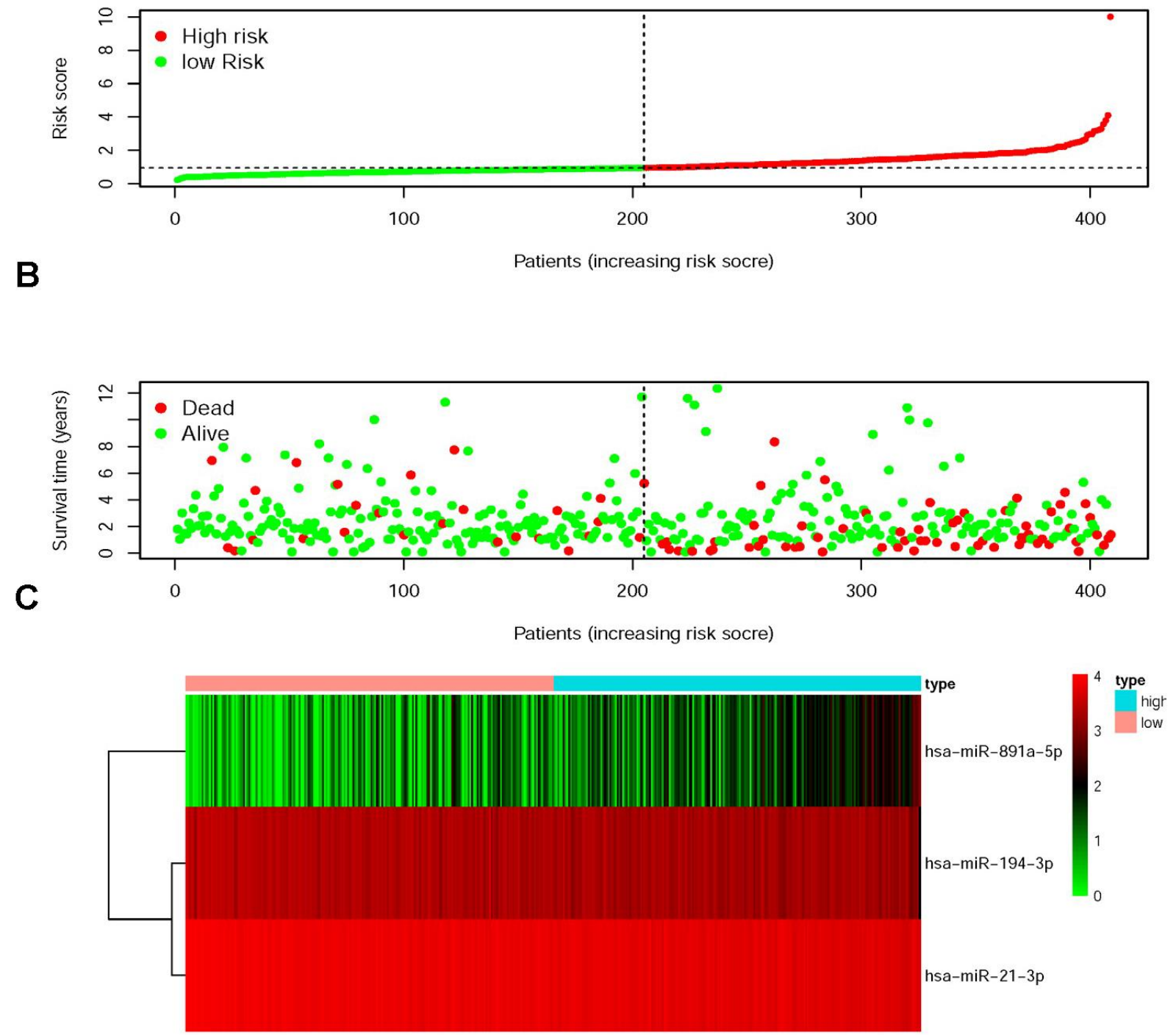

Figure 3. Risk score model (RSM) was established based on SDMIRs. The distribution of risk score in the high-risk group and the lowrisk group (A). Survival status in the low-risk group and the high-risk group (B). The heatmap of the expression levels of sDMIRs (hsa-miR-194$3 p$, hsa-miR-21-3p and hsa-miR-891a-5p) contained in the RSM (C) based on TCGA database. 
miRTarBase and miRDB (Figure 7A-7C). Meanwhile, Figure 7D illustrated the regulatory net among the sDMIRs and TGs. Additionally, we utilized the GO and KEGG analyses to investigate the related molecular mechanisms of these TGs. We found that, in the biological processes, cellular components and molecular

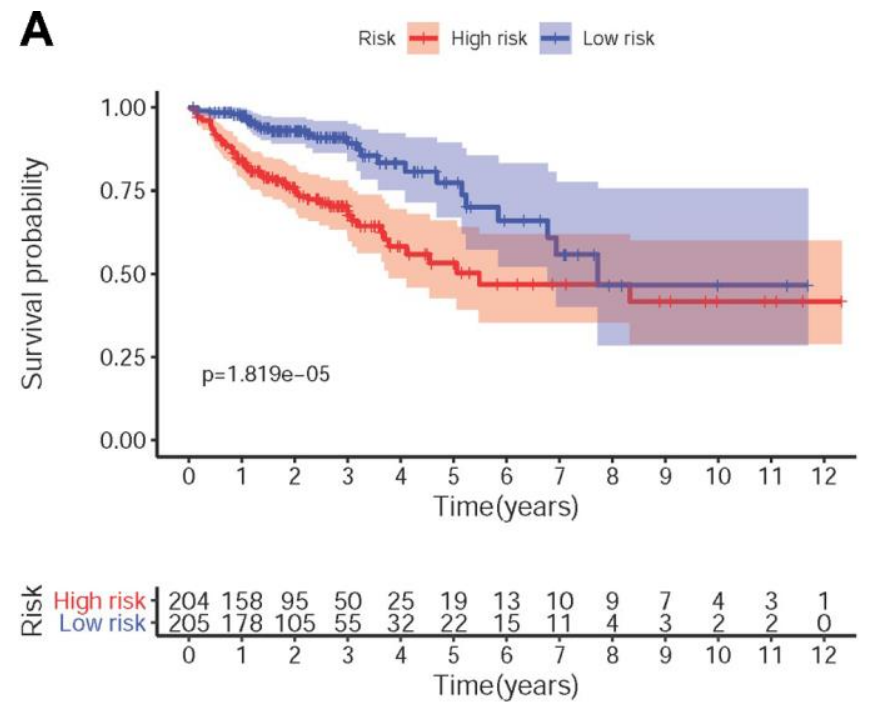

function, "cell morphogenesis involved in neuron differentiation", "adherens junction" and "transcription coregulator activity" were the most significant enrichments, respectively (Figure 8A). In KEGG analysis, "Herpes simplex virus 1 infection" was the most enriched (Figure 8B).

B

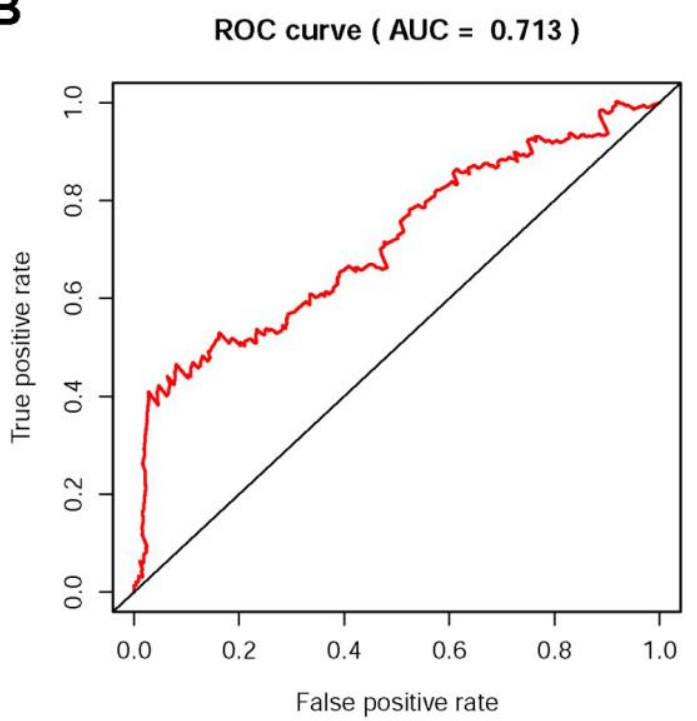

Figure 4. The survival curve and receiver operating characteristic (ROC) curve of RSM. Kaplan-Meier survival curve of OS in the high-risk group and the low-risk group (A). The high-risk group showed the poor prognoses in the CRC patients based on TCGA database. The ROC curve relevance the accuracy of the RSM (B) and the area under curve (AUC) was 0.713 based on TCGA database.

A
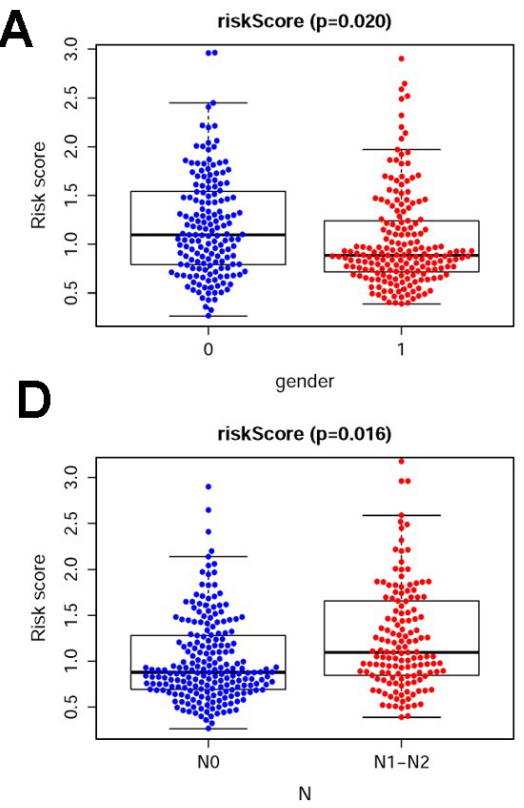
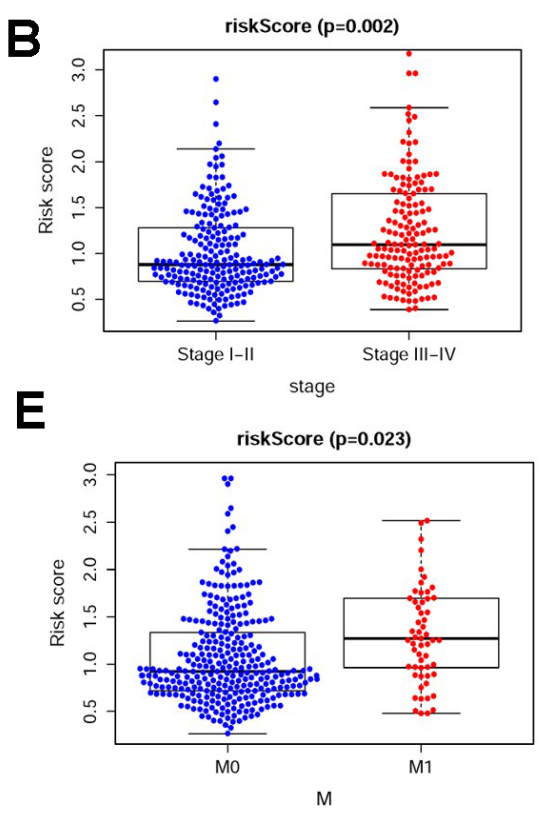

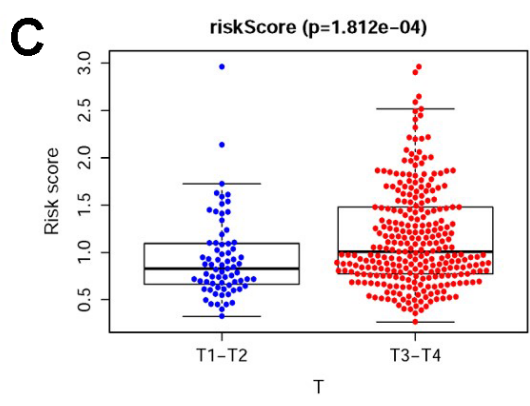

Figure 5. The relevancies between the clinical features and the RSM. The relevancies between the RSM and gender (A), stage (B), Tstage (C), N-stage (D) and M-stage (E). The lower risk scores were showed in the male patients (A), and patients with early stage (B), early Tstage (C), early N-stage (D) and early M-stage (E) based on TCGA database. ( $0=$ Female patients; 1 = Male patients). 
A

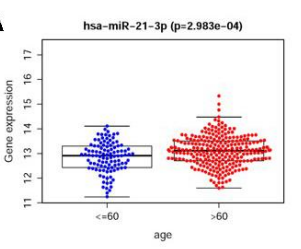

$\mathbf{F}$

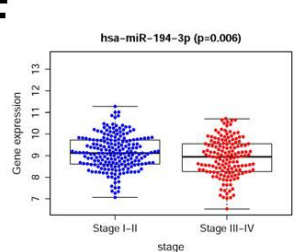

B

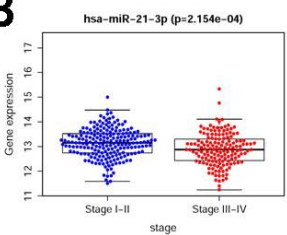

G

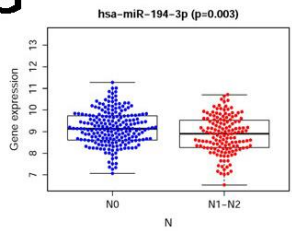

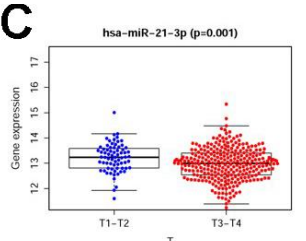
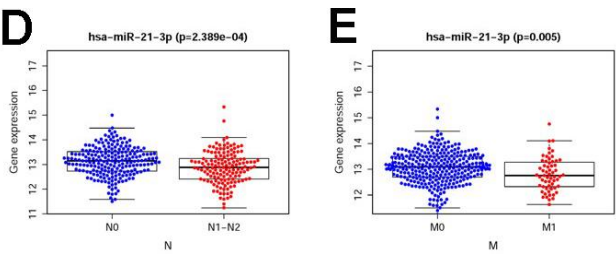

H

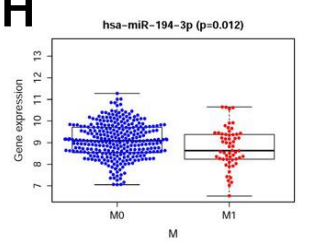

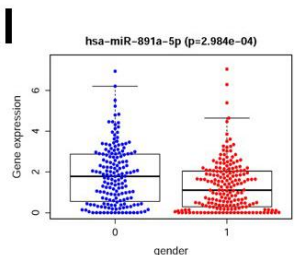

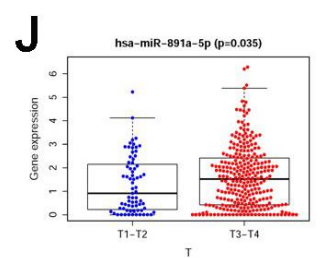

Figure 6. The relevancies between the clinical features and sDMIRs. The expression levels of hsa-miR-21-3p were increased in older patients (A), patients with early stage (B), early T-stage (C), early N-stage (D) and early M-stage (E). The expression levels of hsa-miR-194-3p were decreased in the patients with advanced stage $(\mathbf{F})$, advanced $\mathrm{N}$-stage $(\mathbf{G})$ and advanced $\mathrm{M}$-stage $(\mathbf{H})$. The female patients $(\mathbf{I})$ and patients with advanced T-stage $(\mathrm{J})$ were correlated with the higher expression levels of hsa-miR-891a-5p based on TCGA database. $(0=$ Female patients; 1 = Male patients).

A

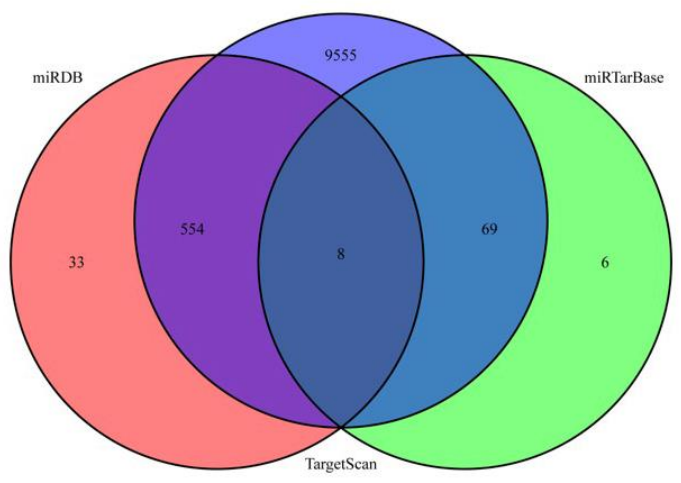

C

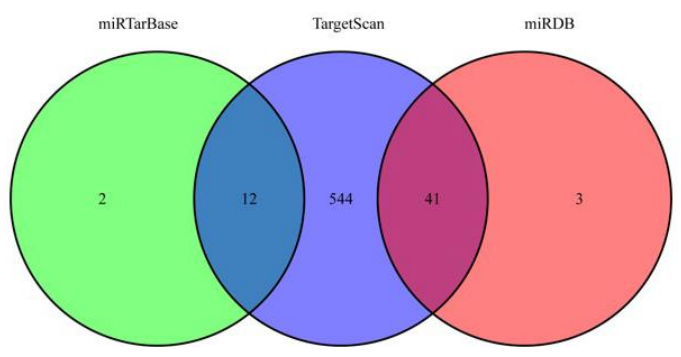

B hsa-miR-194-3p

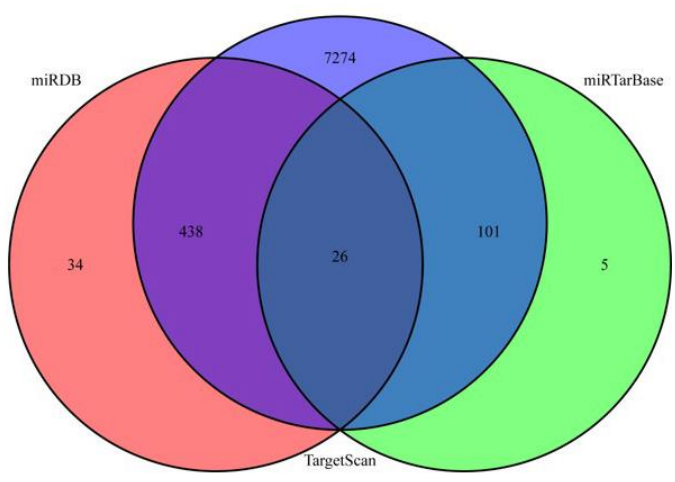

D

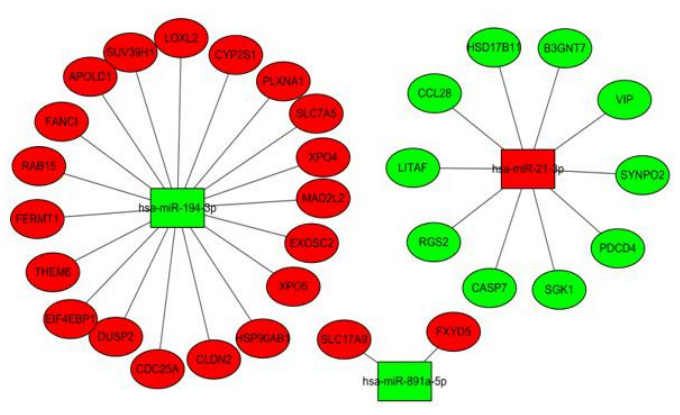

Figure 7. The Venn diagram of the sDMIRs TGs and the regulatory networks of sDMIRs. The predicted TGs were screened from miRDB, TargetScan, and miRTarBase databases. The overlaps meant the numbers of TGs predicted by more than one database. ((A) hsa-miR21-3p; (B) hsa-miR-194-3p; (C) hsa-miR-891a-5p). The regulatory networks of sDMIRs and TGs (D); green parts represent down-regulation and red parts represent up-regulation. 


\section{MiR-21-3p, miR-194-3p and miR-891a-5p obtained the prominent clinical significance}

To further verify the clinical significance of the RSM, we tested the expression and clinical relevance of miR21-3p, miR-194-3p and miR-891a-5p which were involved in the RSM in vivo and in vitro. As showed in Figure 9, miR-21-3p (Figure 9A) and miR-194-3p
(Figure 9B) expressed lowly in CRC cell lines than that in the colonic epithelial cell, but miR-891a-5p (Figure 9C) showed the reversed results. Additionally, we investigated the relevance of the three sDMIRs with Tstage. We found that, compared to adjacent tissues, the expression of miR-891a-5p rather than miR-21-3p and miR-194-3p was higher in carcinoma tissue (Figure 9D). Additionally, the gradually higher expression of
A

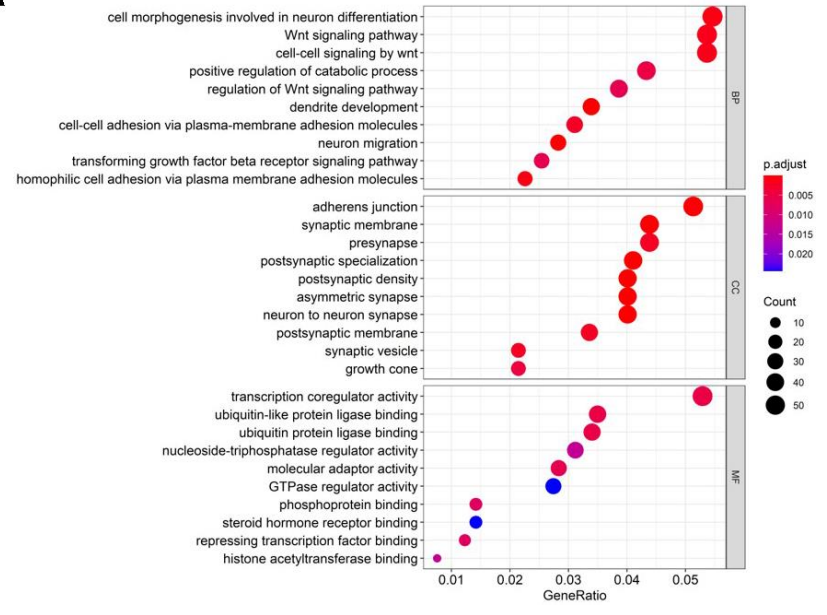

B

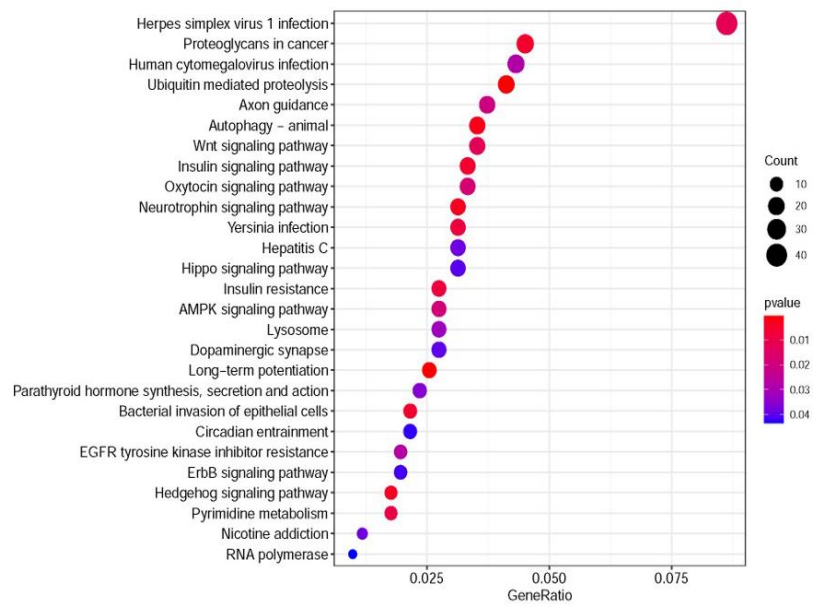

Figure 8. The functional enrichment analysis of TGs. The top pathways of TGs were demonstrated in biological process (BP), cellular component (CC), molecular function (MF) (A), and KEGG pathway (B).

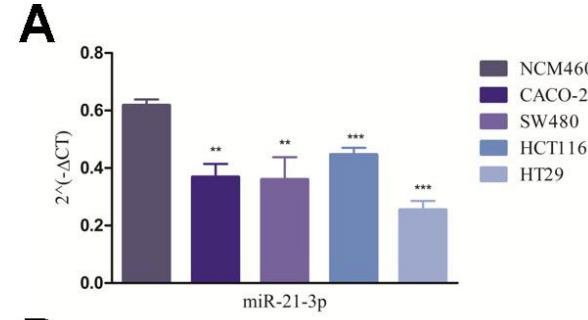

D

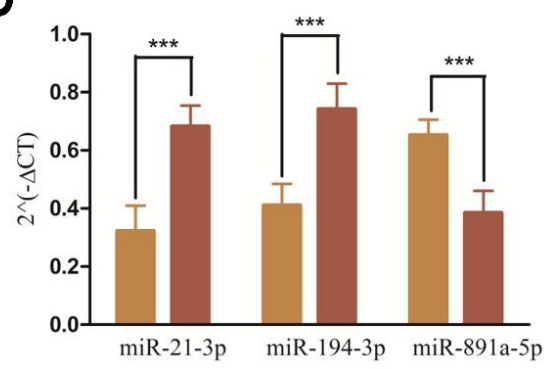

B

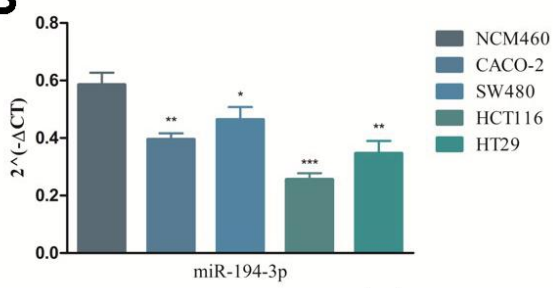

E

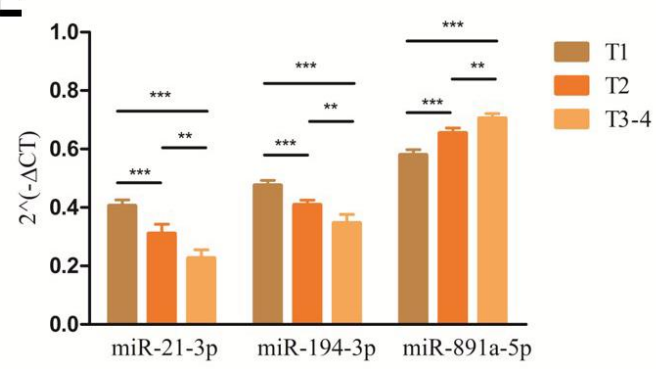

Figure 9. The expression levels of miR-21-3p, miR-194-3p and miR-891a-5p in patients with CCa and its correlation with Tstages. The results of RT-qPCR of miR-21-3p' (A), miR-194-3p' (B) and miR-891a-5p' (C) expression levels in colonic cancer cell lines. ${ }^{* * *},{ }^{* *}$ and * represent the remarkable difference compared with NCM460 $(P<0.001, P<0.01$ and $P<0.05)$, respectively. The expression levels of miR21-3p, miR-194-3p and miR-891a-5p in carcinoma tissues and adjacent tissues based on the CRC samples of Chongqing University Cancer Hospital Clinical (D). ${ }^{* * *}$ Represent the significant difference compared with adjacent tissues $(P<0.001)$. The expression levels of miR-21-3p, miR-194-3p and miR-891a-5p in CCa tissues with various T-stages based on the CRC samples of Chongqing University Cancer Hospital Clinical (E). ${ }^{* * *}, * *$ and $*$ represent the significant difference between groups $(P<0.001, P<0.01$ and $P<0.05)$, respectively. 
miR-891a-5p was tested in tumor tissues of CRC patients with more advanced T-stage, and miR-21-3p and miR-194-3p expressed decreasingly as the more advanced T-stages (Figure 9E).

\section{DISCUSSION}

Recently, accumulating miRNAs were validated to play pivotal roles on diagnosis and prediction in the field of cancer. Gmerek detected a cascade of 40 miRNAs with differential expression in cancerous, of which 8 miRNAs could be used as potential biomarkers for diagnosis of CRC patients [22]. Tang found miRNA$320 \mathrm{~d}$ in tumor-derived exosome could be utilized as a marker for metastatic CRC [23]. Given the values of miRNAs on diagnosis and prognosis prediction, discovering more promising and sensitive biomarkers such as DMIRs attracted increasing attentions.

In the past few years, increasing studies illuminated the crucial roles of TME in the prognoses of patients. Hinshaw found the TME innately modulates cancer progression [24]. Therefore, more researchers began to turn their eyes on the roles of TME. Besides, large quantities of studies have identified various DMIRs with the significant assessing potential for CRC [3, 25, 26]. Although amounts of studies of various cancers have elucidated the clinical significance of DMIRs, especially in tumor invasion, metastasis and prognosis, the complete genome-wide analysis of CRC remain insufficiency, especially on the mechanisms [27-29]. Sun highlighted that miR-21-3p could promote the progression of gastric cancer [13]. Bruna Calsina revealed that miR-21-3p, as a biomarker, was potential for risk stratification to improve the management of patients with pheochromocytomas and paragangliomas [14]. Besides, miR-194-3p has been demonstrated to stimulate the occurrence and progression of breast cancer [17]. However, the roles of miR-21-3p, miR194-3p and miR-891a-5p in predicting prognosis of CRC remain unclear. It has been reported that miR-21$3 p$ promoted cell proliferation, migration and invasion through regulating TSC2/mTOR pathway, and augmented tumor proliferation via PI3K/AKT pathway $[14,30]$. MiR-194-3p strengthened cell proliferation, and migration through targeting and regulating the protein regulator of cytokinesis-1 and methyl $\mathrm{CpG}$ binding protein-2 $[17,19]$. MiR-891a-5p could inhibited tumor cell proliferation, migration and invasion by NFkB pathway [31].

In the present study, we analyzed the expression of mRNAs and miRNAs in 437 CRC patients in TCGA. Next, we identified 3 sDMIRs used to establish the RSM, in which the high-risk group had the poorer OS. The female and advanced stage and TNM-stage got the higher risk scores in RSM. Although female CRC patients have a lower incidence than male CRC patients, the morbidity and mortality ratio of female patients is slightly higher than that of male patients. Hence, this may be the reason why women have higher risk score than men [32]. We established the regulation networks of TGs and three sDMIRs to find the TGs of three sDMIRs. Besides, we analyzed the TGs by the KEGG and GO analysis to detect the potential molecular mechanisms. In KEGG analysis, "Herpes simplex virus 1 infection" was the most enriched. In the biological processes, cellular components and molecular function, "cell morphogenesis involved in neuron differentiation", "adherens junction" and "transcription coregulator activity" were the most significant enrichments, respectively. These results of mechanisms need to be verified by more experiments in the future.

In order to enhance the reliability and persuasion of our RSM, we recruited some patients with CRC. We detected the expression of sDMIRs with obvious clinical significances in CRC clinical samples. The expression of miR-891a-5p was lower in adjacent tissues than that in carcinoma tissues, but miR-21-3p and miR-194-3p expressed highly in adjacent tissues. Besides, the higher expression of miR-891a-5p was related to more advanced T-stage, but miR-21-3p and miR-194-3p showed the reversed results.

Although we demonstrated the clinical significance of RSM in assessing prognoses of CRC patients and tested the expression of sDMIRs involved in RSM, certain limitations are still needed to be improved in future studies. First, multiple omics analysis should be conducted to better ascertain the significance and mechanisms of sDMIRs. Then, in vivo and in vitro detections of other sDIMRs involved in RSM should also be completed.

\section{CONCLUSIONS}

In this study, we demonstrated the clinical significance of the RSM which was established base on sDMIRs, especially in assessing prognoses of CRC patients. These findings open up new avenues for clinical decisions and provide an accurate and novel model for prognostic evaluation of CRC patients.

\section{Abbreviations}

CCa: colon cancer; CRC: colorectal cancer; DMIRs: differentially expressed miRNAs; RSM: risk score model; sDMIRs: survival-related differentially expressed miRNAs; OS: overall survival; TCGA: The Cancer Genome Atlas; TME: tumor microenvironment. 


\section{AUTHOR CONTRIBUTIONS}

Lumi Huang, Yiming Wang and Hao Long contributed to the research design. Yiming Wang and Nan Shan wrote the paper. Huiwen Ma and Hao long contributed to the data verification and analysis. Xingyue Chen and Songmei Lu collected clinical samples.

\section{CONFLICTS OF INTEREST}

The authors declare that they have no conflicts of interest.

\section{FUNDING}

Chongqing Science and Technology Bureau, cstc2019jcyj-msxmX0721.

\section{REFERENCES}

1. Angius A, Uva P, Pira G, Muroni MR, Sotgiu G, Saderi L, Uleri E, Caocci M, Ibba G, Cesaraccio MR, Serra C, Carru $C$, Manca $A$, et al. Integrated Analysis of miRNA and mRNA Endorses a Twenty miRNAs Signature for Colorectal Carcinoma. Int J Mol Sci. 2019; 20:4067. https://doi.org/10.3390/ijms20164067 PMID:31434359

2. Sánchez-Hidalgo JM, Rodríguez-Ortiz $L$, Arjona-Sánchez Á, Rufián-Peña S, Casado-Adam Á, Cosano-Álvarez A, Briceño-Delgado J. Colorectal peritoneal metastases: Optimal management review. World J Gastroenterol. 2019; 25:3484-502.

https://doi.org/10.3748/wjg.v25.i27.3484

PMID:31367152

3. Chen B, Xia Z, Deng YN, Yang Y, Zhang P, Zhu H, Xu N, Liang S. Emerging microRNA biomarkers for colorectal cancer diagnosis and prognosis. Open Biol. 2019; 9:180212.

https://doi.org/10.1098/rsob.180212

PMID:30958116

4. Sillo TO, Beggs AD, Morton DG, Middleton G. Mechanisms of immunogenicity in colorectal cancer. $\mathrm{Br}$ J Surg. 2019; 106:1283-97.

https://doi.org/10.1002/bjs.11204

PMID:31216061

5. de Miguel Pérez D, Rodriguez Martínez A, Ortigosa Palomo A, Delgado Ureña M, Garcia Puche JL, Robles Remacho A, Exposito Hernandez J, Lorente Acosta JA, Ortega Sánchez FG, Serrano MJ. Extracellular vesiclemiRNAs as liquid biopsy biomarkers for disease identification and prognosis in metastatic colorectal cancer patients. Sci Rep. 2020; 10:3974.

https://doi.org/10.1038/s41598-020-60212-1

PMID:32132553
6. Fadaka AO, Pretorius A, Klein A. Biomarkers for Stratification in Colorectal Cancer: MicroRNAs. Cancer Control. 2019; 26:1073274819862784.

https://doi.org/10.1177/1073274819862784 PMID:31431043

7. Hernández R, Sánchez-Jiménez E, Melguizo C, Prados J, Rama AR. Downregulated microRNAs in the colorectal cancer: diagnostic and therapeutic perspectives. BMB Rep. 2018; 51:563-71.

https://doi.org/10.5483/BMBRep.2018.51.11.116 PMID:30158023

8. Ju J. miRNAs as biomarkers in colorectal cancer diagnosis and prognosis. Bioanalysis. 2010; 2:901-06. https://doi.org/10.4155/bio.10.45 PMID:20543966

9. Ke MJ, Ji LD, Li YX. Explore prognostic marker of colorectal cancer based on ceRNA network. J Cell Biochem. 2019; 120:19358-70. https://doi.org/10.1002/jcb.28860 PMID:31490563

10. Yang Y, Qu A, Wu Q, Zhang X, Wang L, Li C, Dong Z, Du $L$, Wang C. Prognostic value of a hypoxia-related microRNA signature in patients with colorectal cancer. Aging (Albany NY). 2020; 12:35-52.

https://doi.org/10.18632/aging.102228

PMID:31926112

11. Soleimani A, Rahmani F, Saeedi N, Ghaffarian R, Khazaei M, Ferns GA, Avan A, Hassanian SM. The potential role of regulatory microRNAs of RAS/MAPK signaling pathway in the pathogenesis of colorectal cancer. J Cell Biochem. 2019; 120:19245-53. https://doi.org/10.1002/jcb.29268 PMID:31512778

12. Sun $M$, Song $H$, Wang $S$, Zhang $C$, Zheng $L$, Chen $F$, Shi D, Chen Y, Yang C, Xiang Z, Liu Q, Wei C, Xiong B. Integrated analysis identifies microRNA-195 as a suppressor of Hippo-YAP pathway in colorectal cancer. J Hematol Oncol. 2017; 10:79.

https://doi.org/10.1186/s13045-017-0445-8 PMID:28356122

13. Sun X, Zhang K, Li D. Prognostic potential of miR-21-3p in gastric cancer. J BUON. 2020; 25:2678-82. PMID: $\underline{3455113}$

14. Calsina $B$, Castro-Vega $L$, Torres-Pérez $R$, Inglada-Pérez $L$, Currás-Freixes $M$, Roldán-Romero JM, Mancikova V, Letón R, Remacha L, Santos M, Burnichon N, LusseyLepoutre C, Rapizzi E, et al. Integrative multi-omics analysis identifies a prognostic miRNA signature and a targetable miR-21-3p/TSC2/mTOR axis in metastatic pheochromocytoma/paraganglioma. Theranostics. 2019; 9:4946-58.

https://doi.org/10.7150/thno.35458 PMID: $\underline{31410193}$

15. Gao Z, Liu H, Shi Y, Yin L, Zhu Y, Liu R. Identification of Cancer Stem Cell Molecular Markers and Effects of hsa- 
miR-21-3p on Stemness in Esophageal Squamous Cell Carcinoma. Cancers (Basel). 2019; 11:518.

https://doi.org/10.3390/cancers11040518

PMID:30979011

16. Edvardsson K, Nguyen-Vu T, Kalasekar SM, Pontén F, Gustafsson JÅ, Williams C. Estrogen receptor $\beta$ expression induces changes in the microRNA pool in human colon cancer cells. Carcinogenesis. 2013; 34:1431-41.

https://doi.org/10.1093/carcin/bgt067

PMID:23436804

17. Zhou Q, Guo J, Huang W, Yu X, Xu C, Long X. LincROR promotes the progression of breast cancer and decreases the sensitivity to rapamycin through miR194-3p targeting MECP2. Mol Oncol. 2020; 14:2231-50.

https://doi.org/10.1002/1878-0261.12700

PMID:32335998

18. Kubo H, Hiroshima $Y$, Mori R, Saigusa $Y$, Murakami T, Yabushita Y, Sawada Y, Homma Y, Kumamoto T, Matsuyama R, Endo I. MiR-194-5p in Pancreatic Ductal Adenocarcinoma Peritoneal Washings is Associated with Peritoneal Recurrence and Overall Survival in Peritoneal Cytology-Negative Patients. Ann Surg Oncol. 2019; 26:4506-14.

https://doi.org/10.1245/s10434-019-07793-y PMID:31489551

19. Yi L, Ouyang L, Wang S, Li SS, Yang XM. Long noncoding RNA PTPRG-AS1 acts as a microRNA-194-3p sponge to regulate radiosensitivity and metastasis of nasopharyngeal carcinoma cells via PRC1. J Cell Physiol. 2019; 234:19088-102.

https://doi.org/10.1002/jcp.28547 PMID:30993702

20. Zhang Z, Xu L, He L, Wang J, Shi X, Li Z, Shi S, Hou K, Teng Y, Qu X. MiR-891a-5p as a prognostic marker and therapeutic target for hormone receptor-positive breast cancer. J Cancer. 2020; 11:3771-82.

https://doi.org/10.7150/ica.40750

PMID:32328182

21. Liu Y, Gou X, Wei Z, Yu H, Zhou X, Li X. Bioinformatics profiling integrating a four immune-related long noncoding RNAs signature as a prognostic model for papillary renal cell carcinoma. Aging (Albany NY). 2020; 12:15359-73.

https://doi.org/10.18632/aging.103580

PMID:32716909

22. Gmerek L, Martyniak K, Horbacka K, Krokowicz P, Scierski W, Golusinski P, Golusinski W, Schneider A, Masternak MM. MicroRNA regulation in colorectal cancer tissue and serum. PLoS One. 2019; 14:e0222013.

https://doi.org/10.1371/journal.pone.0222013

PMID:31469874
23. Tang $Y$, Zhao $Y$, Song $X$, Song $X$, Niu L, Xie L. Tumorderived exosomal miRNA-320d as a biomarker for metastatic colorectal cancer. J Clin Lab Anal. 2019; 33:e23004.

https://doi.org/10.1002/jcla.23004 PMID:31420913

24. Hinshaw DC, Shevde LA. The Tumor Microenvironment Innately Modulates Cancer Progression. Cancer Res. 2019; 79:4557-66.

https://doi.org/10.1158/0008-5472.CAN-18-3962 PMID: $\underline{1350295}$

25. Kijima T, Hazama S, Tsunedomi R, Tanaka H, Takenouchi H, Kanekiyo S, Inoue $\mathrm{Y}$, Nakashima M, lida M, Sakamoto K, Suzuki N, Takeda S, Ueno T, et al. MicroRNA-6826 and -6875 in plasma are valuable noninvasive biomarkers that predict the efficacy of vaccine treatment against metastatic colorectal cancer. Oncol Rep. 2017; 37:23-30.

https://doi.org/10.3892/or.2016.5267

PMID:27878288

26. To KK, Tong CW, Wu M, Cho WC. MicroRNAs in the prognosis and therapy of colorectal cancer: From bench to bedside. World J Gastroenterol. 2018; 24:2949-73.

https://doi.org/10.3748/wig.v24.i27.2949

PMID: $\underline{30038463}$

27. Bertoli G, Cava C, Castiglioni I. MicroRNAs: New Biomarkers for Diagnosis, Prognosis, Therapy Prediction and Therapeutic Tools for Breast Cancer. Theranostics. 2015; 5:1122-43. https://doi.org/10.7150/thno.11543 PMID:26199650

28. Kosaka N, Iguchi H, Ochiya T. Circulating microRNA in body fluid: a new potential biomarker for cancer diagnosis and prognosis. Cancer Sci. 2010; 101:2087-92.

https://doi.org/10.1111/j.1349-7006.2010.01650.x PMID:20624164

29. Nassar FJ, Nasr R, Talhouk R. MicroRNAs as biomarkers for early breast cancer diagnosis, prognosis and therapy prediction. Pharmacol Ther. 2017; 172:34-49. https://doi.org/10.1016/i.pharmthera.2016.11.012 PMID:27916656

30. Zhu Y, Tang H, Zhang L, Gong L, Wu G, Ni J, Tang X. Suppression of miR-21-3p enhances TRAIL-mediated apoptosis in liver cancer stem cells by suppressing the PI3K/Akt/Bad cascade via regulating PTEN. Cancer Manag Res. 2019; 11:955-68.

https://doi.org/10.2147/CMAR.S183328 PMID: $\underline{0774424}$

31. Yao S, Hu M, Hao T, Li W, Xue X, Xue M, Zhu X, Zhou F, Qin D, Yan $Q$, Zhu J, Gao SJ, Lu C. MiRNA-891a-5p mediates HIV-1 Tat and KSHV Orf-K1 synergistic 
induction of angiogenesis by activating NF-KB signaling. Nucleic Acids Res. 2015; 43:9362-78.

https://doi.org/10.1093/nar/gkv988 PMID:26446987

32. Bray F, Ferlay J, Soerjomataram I, Siegel RL, Torre LA, Jemal A. Global cancer statistics 2018: GLOBOCAN estimates of incidence and mortality worldwide for 36 cancers in 185 countries. CA Cancer J Clin. 2018; 68:394-424.

https://doi.org/10.3322/caac.21492

PMID:30207593 


\section{SUPPLEMENTARY MATERIALS}

\section{Supplementary Figure}
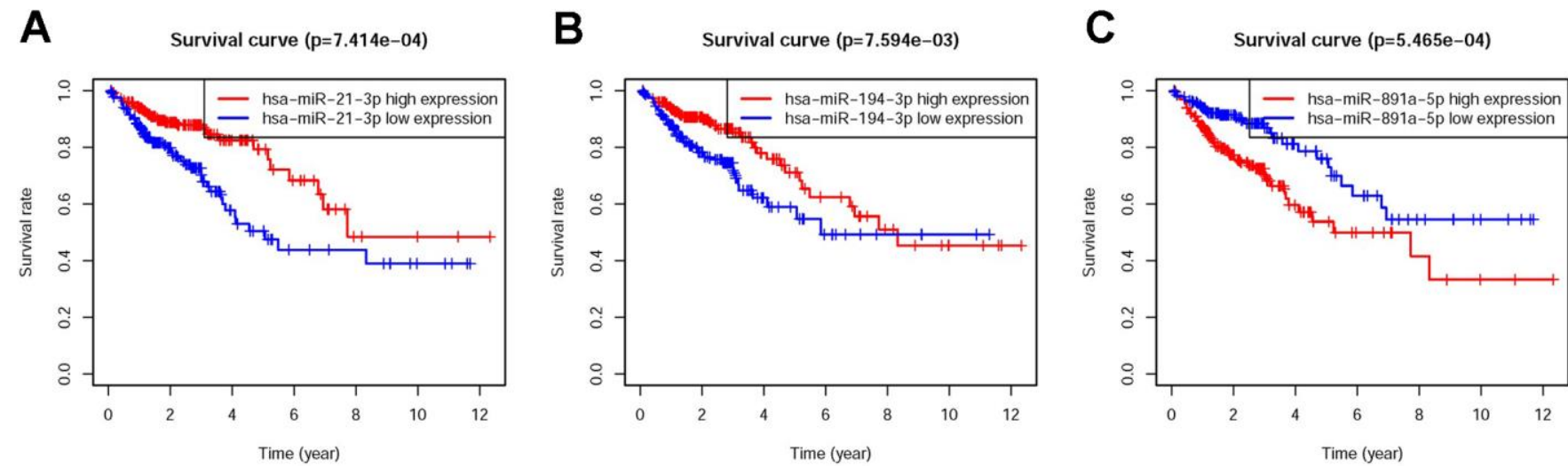

Supplementary Figure 1. Survival curve of sDMIRs. Kaplan-Meier survival curve of sDMIRs in CRC patients. The higher expression levels of hsa-miR-21-3p (A) and hsa-miR-194-3p (B) were correlated with the longer OS based on TCGA database. The higher expression levels of hsa-miR-891a-5p (C) were related with the poor prognosis based on TCGA database. 


\section{Supplementary Table}

Please browse Full Text version to see the data of Supplementary Table 1.

Supplementary Table 1. The expression information of 496 DMIRs. 